811.131.1:811.163.41

https://doi.org/10.18485/italbg.2021.1.8

\author{
Rita Scotti Jurić* \\ Università "Juraj Dobrila" di Pola \\ Lorena Lazaric ${ }^{* *}$ \\ Università "Juraj Dobrila" di Pola
}

\title{
GIANNI RODARI: PAROLE PER PENSARE, PAROLE PER AMARE
}

\begin{abstract}
I dilemmi che da sempre tormentano coloro che si occupano di traduzione poetica possono venir riassunti in due quesiti di base: il primo, di natura prevalentemente filosofica, se è possibile tradurre la poesia, e il secondo, di natura pratica, come tradurre un testo poetico (Campanini 2001). Questo saggio racchiude un tentativo di traduzione poetica dall'italiano al croato di alcune filastrocche/poesie di Rodari accompagnato da riflessioni operative, senza la pretesa di proporre un modello ideale anche perché "la bontà di una traduzione non può stabilirsi a priori" (Praz 1925: 10) ma può avere solo una validità contingente, legata ai gusti dell'epoca oppure ai valori estetici e ideologici del singolo traduttore. Ciò significa che più che il plauso della critica, sarà il consenso del pubblico vasto ed eterogeneo a esprimere un giudizio di accoglienza o di rifiuto. La dinamica delle operazioni linguistiche e concettuali che hanno generato la versione definitiva delle presenti traduzioni è stata molto complessa e discussa considerando che nell'effettuare le singole scelte traduttologiche è stato necessario tenere conto simultaneamente di molteplici e diversi fattori nella loro interazione: contenuto, ritmo, rime, assonanze, significati, costumi, cultura.

Parole chiave: Gianni Rodari, traduzione poetica, globalità, località, cultura e tradizione.
\end{abstract}

\section{INTRODUZIONE}

Gianni Rodari, scrittore e giornalista italiano (Omegna, Lago d'Orta, 23 ottobre 1920 - Roma, 14 aprile 1980), di professione faceva l'insegnante. Dopo il 1944 diresse i giornali Ordine Nuovo e Pioniere e collaborò al quotidiano L'Unità. Ricorrono cento anni dalla sua nascita, un numero che lascia incredulo chi lo conosce attraverso i suoi libri, storie moderne incarnate in una forma "classica": universale, eterna, perfetta. Le innumerevoli storie che compongono l'opera rodariana sono una fonte inesauribile di

\footnotetext{
*rscotti@unipu.hr

**1orena.lazaric@unipu.hr
} 
creatività e con una semplicità geniale raccontano, da decenni, una realtà sempre attuale e ispirano un'attività che allo scrittore stava molto a cuore: il gioco. Il gioco linguistico, quello fantastico, trova grande spazio nei suoi racconti e fa parte delle tante forme che Rodari ha saputo dare alla fantasia, tramite invenzioni che sono e rimarranno insuperabili. Le sue raccolte di storie diventano un piccolo tesoro per i lettori di ieri e di oggi, e una grande sorpresa che attende quelli di domani.

"Le favole dove stanno? // C'è n'è una in ogni cosa: // nel legno del tavolino, // nel bicchiere, nella rosa." (Rodari 1972: 137), recita l'autore nella filastrocca La bella addormentata della raccolta Filastrocche in cielo $e$ in terra. Rivoluzionaria, perché al posto dei protagonisti avventurosi ed esotici porta alla ribalta artigiani, operai, modesti impiegati, emigranti, padri di famiglia costretti a combattere con magri stipendi. Illuminati dalla luce della poesia essi rivelano le meraviglie del quotidiano, delle piccole gioie domestiche, delle grandi speranze e dei grandi ideali: la fratellanza universale, l'integrazione raziale, la non violenza. La cooperazione e la solidarietà prendono il posto della competizione e il potere del denaro perde ogni incanto. "Con divertimento l'autore dissacra la matita rossa e blu, ridimensiona gli errori degli scolari, gioca con le parole, introduce il nonsense, capovolge i proverbi e pone le basi d'un modo nuovo d'essere uomini e di vivere l'infanzia" (Buongiorno 1995: 125). Rodari ha la capacità di parlare di cose serissime con la levità nell'aria, l'ironia e la leggerezza (Boero 2007). Nella sua completezza, egli scrive rivolgendosi ai piccoli ma con l'intento di farsi capire anche dai grandi muovendosi fra la fantasia e la realtà che ci circonda in quanto sa che il mondo della fiaba non finisce con il trapasso del bambino dalla prima infanzia alla fanciullezza. Scrive fiabe e racconti "legati a una fantasia incredibilmente sfrenata, ma che collega secondo la 'logica' del bambino strane cose, strani avvenimenti, delle associazioni che un adulto rifiuterebbe, ma che per il bambino sono reali" (Denti 2014: 75-76). Le sue fiabe sono calate nel mondo che circonda il bambino, nella società, con l'intento di stimolare l'interesse del lettore per il mondo dell'oggi, di quello che è chiamato il sociale, il pubblico, oltre che per la vita privata.

\section{LE TRADUZIONI DI GIANNI RODARI NEL MONDO}

Prima di discutere delle problematiche traduttive in generale è doverosa una lettura critica della fortuna di Rodari all'estero: infatti, i libri da lui scritti negli anni cinquanta hanno avuto grande diffusione specialmente nell'Est europeo, qualche anno dopo in America Latina, Asia e Australia, mentre dal 
2002 le sue opere sono approdate anche negli Stati Uniti. In tal modo sono apparse traduzioni nelle più svariate lingue del mondo: dal giapponese al lituano, dallo slovacco allo sloveno, dallo spagnolo al cinese, al mongolo, al brasiliano, all'argentino, al vietnamita e poi traduzioni in inglese e francese.

Nel panorama letterario italiano il fenomeno di Gianni Rodari è particolare, in quanto costituisce un raro caso di autore divenuto famoso in patria solo dopo essere stato tradotto all'estero. Si tratta di "effetto di rimbalzo" dovuto all'enorme successo ottenuto innanzitutto in Unione Sovietica. Nel corso degli anni la fortuna della sua produzione è andata crescendo impetuosamente e oggi le sue opere risultano tradotte in cinquantatré lingue e in cinquantacinque Paesi ${ }^{1}$. Le prime traduzioni di Rodari risalgono al 1953, quando Le avventure di Cipollino e Il libro delle filastrocche, i suoi primi due libri, usciti in Italia nel 1951, vengono pubblicati rispettivamente in Bulgaria e in Unione Sovietica, inaugurando il suo grande e rapido successo nei paesi socialisti. Attraverso la lingua russa le opere di Rodari vengono tradotte dapprima, nel 1954, in Cina e Mongolia e poi, tra gli anni Settanta e Ottanta, nelle numerose lingue delle repubbliche sovietiche: uzbeko, altai, kazako, jakuto, georgiano, azero, tagico, armeno e tataro. A queste si aggiungono il lituano, l'ucraino, l'estone e il lettone. Le avventure di Cipollino, in cui il protagonista guida la rivolta pacifica contro l'oppressore, si afferma come la sua opera più popolare: nel 1954 viene pubblicata in Polonia, Germania dell'Est e Cecoslovacchia, successivamente in Ungheria, Albania e infine in Jugoslavia, tradotta nelle diverse lingue della federazione. Di grande popolarità godono anche le opere posteriori, in modo particolare Gelsomino nel paese dei bugiardi, la cui prima traduzione esce nel 1963 in URSS, e Il viaggio della freccia azzurra in Polonia nel 1955.

In Occidente la diffusione di Rodari si afferma molto più tardi rispetto agli Stati oltrecortina, con Avventure di Cipollino in Francia e Giappone nel 1956 e negli anni Sessanta con Le favole al telefono nella Germania federale e nel Regno Unito. Negli anni Ottanta iniziano le numerose traduzioni greche, giapponesi, francesi, spagnole, catalane, basche, galiziane e asturiane. Nel 1970 Rodari vince il premio internazionale Hans Christian Andersen ${ }^{2}$ e negli anni Ottanta le sue opere raggiungono nuovi paesi: i Paesi Bassi (Favole al telefono, 1983), il Portogallo (Le avventure di Cipollino, 1984),

${ }^{1}$ Questi dati sono stati ampiamente reperiti nel saggio di Andrea Palermitano, Gianni Rodari in altre lingue, 14/05/2020. Testo disponibile sul sito https://www.newitalianbooks. it/it/gianni-rodari-nel-mondo/ [Consultato il 15/11/2020].

${ }^{2}$ Rodari è l'unico italiano ad aver ricevuto il premio Hans Cristian Andersen, noto anche come il "Piccolo Premio Nobel" della narrativa per l'infanzia, conferito dal 1956. Testo disponibile sul sito https://it.wikipedia.org/wiki/Premio_Hans_Christian_Andersen [Consultato il 12/1/2020]. 
1'Iran (La torta nel cielo, 1985), la Danimarca e la Norvegia (Grammatica della fantasia, 1987), la Svezia (Grammatica della fantasia, 1988) e la Siria (La torta nel cielo, 1989, prima traduzione in arabo). Nella Federazione Russa le traduzioni si moltiplicano fin dai primi anni Novanta, dal mar Baltico alla Siberia. Più lenta ma costante è la diffusione dei suoi libri in altri ex paesi socialisti come la Bielorussia, la Slovacchia, la Slovenia, la Croazia e la Repubblica Ceca. Ormai entrato saldamente nel panorama internazionale della narrativa per l'infanzia, Grammatica della fantasia è il primo libro edito in Brasile nel 1982 e negli Stati Uniti nel 1995. Nelle Americhe Rodari comincia a la sua fortuna traduttiva solo dopo il Duemila. Negli ultimi decenni le sue opere raggiungono mercati editoriali nuovi, come la Tailandia (Favole al telefono, 1992), la Corea del Sud (Favole al telefono, 1998) e il Vietnam (Le avventure di Cipollino, 2009). Nello stesso periodo i libri di Rodari tornano a circolare in Cina (Palermitano 2020). Le prime traduzioni in tedesco risalgono agli anni Cinquanta e Sessanta, in particolare con Zwiebelchen (Le avventure di Cipollino) e Gutenachtgeschichten am Telefon (Favole al telefono). Negli anni Settanta avviene la pubblicazione di Kopfblumen, una raccolta di filastrocche.

\section{LE TRADUZIONI IN CROAZIA}

L'attività letteraria di Gianni Rodari nasce per caso, con una filastrocca per una bambina di nome Susanna, in seguito pubblicata nell'angolo dedicato ai bambini de L'Unità. In un'altra occasione, su richiesta di una lettrice, pubblicò una filastrocca a suo figlio malato, e da allora non si fermerà più. Divenne famoso come scrittore per bambini, prima con la sua raccolta di poesie Filastrocche in cielo e in terra, 1960 (traduzione croata Brojalice na nebu i na zemlji), e poi romanzi. Il romanzo di Cipollino, 1951 (ristampato nel 1959 come Le avventure di Cipollino, traduzione croata Čipolino), è una finzione allegorica sulla lotta di una piccola nazione contro gli sfruttatori. La narrazione ha anche elementi di una favola perché i personaggi sono frutta e verdura, ma è chiaro che rappresentano le persone. Movimento, immaginazione e chiara espressione degli orientamenti sociali sono le caratteristiche di questa prosa dinamica e avventurosa. L'autore sviluppa anche una storia socialmente impegnata nel suo romanzo Il viaggio della Freccia Azzurra del 1954, dove raffigura una ribellione di giocattoli in fuga dal negozio per bambini poveri. La moltitudine di personaggi mitologici, e alle volte insoliti, che Rodari introduce nella sua prosa apre delle possibilità per una trattazione arguta dei più svariati temi della vita moderna. Anche qui, nella sua narrazione, l'autore usa spesso l'iperbole come elemento espressivo particolarmente efficace. Seguono Gelsomino nel paese dei bugiardi 
(1958), Favole al telefono (1962), La torta in cielo (1966), Il pianeta degli alberi di Natale (1970), Tante storie per giocare (1971) e numerose altre opere. Rodari è un intenditore nel caratterizzare i personaggi che delinea con dialoghi vividi e dettagli interessanti; le loro caratteristiche insolite sono la base per un'espressione convincente della filosofia di vita, dell'etica e dell'atteggiamento nei confronti dei problemi umani. La sua narrazione è intrisa di allegria e sottile umorismo (Zalar 2011). In Croazia, le opere di Rodari vengono tradotte negli anni Sessanta e Settanta, da rinomati traduttori quali Ratko Zvrko, Pavao Pavličić, Ljerka Car-Matutinović e Slobodan Lazić, alcuni dei quali sono anche scrittori. Sono stati pubblicati principalmente dalle case editrici Mladost e Vjeverica, in diverse ristampe, il che dimostra un forte interesse del giovane pubblico croato. L'influenza della sua poetica, in senso di stima e prestigio, ha condizionato parecchie opere di Grigor Vitez, Dubravka Ugrešić e altri narratori fantasy contemporanei.

Di Gianni Rodari sono state tradotte sei opere tra le quali il libro con più pubblicazioni è certamente Le avventure di Cipollino (sei edizioni più una traccia audio per ipo e non vedenti di Ratko Zvrko e due edizioni di Ljerka Car-Matutinović). Un forte interesse hanno destato pure le opere Il pianeta degli alberi di Natale (Planeta ispunjenih želja, tradotto da Pavao Pavličić con cinque edizioni) e Il viaggio della freccia azzurra (Putovanje Plave Strijele, quattro edizioni più una traccia audio per ipo e non vedenti nella traduzione di Ljerka Car-Matutinović e Slobodan Lazić). Favole al telefono (Telefonske priče), Gelsomino nel paese dei bugiardi (Đelsomino u zemlji lažljivaca) hanno avuto un'unica edizione, mentre La torta in cielo (Torta na nebu) ha vissuto una seconda edizione. Dal libro La Freccia Azzurra è stato tratto l'omonimo film d'animazione nel 1996. Il grande successo delle opere di Rodari in Unione Sovietica li ha portati alla realizzazione anche di cartoni animati, come Cipollino (1961), recentemente tradotto e diffuso in Italia per il mercato home video come Rassejannyj Džovanni (1969), tratto da La passeggiata di un distratto . $^{3}$.

In Croazia, il debutto di Rodari in teatro è avvenuto grazie alla Compagnia teatrale di Virovitica con le Favole al telefono (Telefonske priče) nella regia di Dražen Ferenčina, che ha firmato anche la drammaturgia insieme a Draško Zidar, con le scenografie di Mijo Pavelko, i costumi di Mijo Pavelko e Vanda Grba e le musiche di Igor Karlić.

${ }^{3}$ Testo disponibile sul sito https://it.wikipedia.org/wiki/Gianni_Rodari [Consultato il $23 / 10 / 2020]$. 


\section{TEORIE, CORPUS E METODOLOGIA DELLA RICERCA}

Dopo un'aggiornata rassegna della fortuna traduttiva di Gianni Rodari nel mondo, siamo passati all'individuazione e alla catalogazione delle traduzioni in Croazia a partire dalle primissime traduzioni del 1960 fino a oggi. Il valore di tali traduzioni è argomentabile dal fatto che la letteratura per l'infanzia costituisce uno dei primi strumenti espressivi dei giovani lettori ed è la base della loro comprensione del mondo (Reynolds 2011). Le storie raccontate sono una fonte inesauribile di immagini, vocaboli, comportamenti, strutture e spiegazioni necessarie per fare esperienza (Garavini 2014). Tenendo presente questa necessità intellettuale e didattica abbiamo voluto dare il nostro apporto traducendo alcune poesie non conosciute al giovane pubblico croato. Il compito non è stato per niente facile in quanto, pur conservando la sua identità storica, il metatesto ha assunto un'altra identità, nella quale il testo originale è sempre riconoscibile e vivo (Formicola 2015). Partendo dal presupposto che un'opera per bambini, per raggiungere un pubblico più ampio e avere successo, deve rivolgersi a due destinatari differenti, il pubblico primario e il lettore adulto (Shavit 1980), ne consegue che abbiamo dovuto tener conto di ambedue i livelli di espressione (Ewers 2009). Pertanto la ricerca si prefigge di dimostrare che nella traduzione di opere per l'infanzia gli elementi culturali subiscono un alto numero di manipolazioni. Il nostro obiettivo è quello di fornire una traduzione di alta qualità, cosa generalmente trascurata nell'ambito della traduzione per l'infanzia (Scotellaro 2020), attraverso un'armonizzazione terminologica in lingua croata che potrà far conoscere la letteratura per l'infanzia italiana in Croazia. L'analisi mira dunque a individuare quali tipi di "shift" (Garavini 2014) si verificano durante il trasferimento di elementi culturali specifici dal sistema di partenza a quello di arrivo e quali sono le principali strategie utilizzate al fine di assicurare una maggiore leggibilità dei testi tradotti.

Il taglio teorico dal quale si è partiti per accedere all'analisi dei testi si basa sulla Skopostheorie, di Katharina Reiß e Hans J. Vermeer (1984), che sposta l'enfasi sull'equivalenza e sulla fedeltà al testo di partenza verso approcci descrittivi, incentrati sugli scopi della traduzione, sulle funzioni e sullo status della cultura di arrivo. Il concetto centrale alla base di questa teoria è lo skopós, ovvero "purpose, aim, goal, finality, objective; intention" (Vermeer 1996: 4), lo scopo o la funzione della traduzione che rappresenta il fattore decisivo di un progetto traduttivo. Una traduzione "funziona" nel sistema di arrivo se soddisfa le aspettative del lettore del testo di arrivo alla forma testuale e alla funzione (Vermeer 1996). Christiane Nord (1991) aggiunge allo skopos il concetto di "loyalty" con il quale definisce la responsabilità del traduttore sia verso il mittente del testo di partenza che verso il destinatario 
del testo di arrivo. Questo principio morale implica il non voler deludere le convenzioni e le aspettative dei fruitori della traduzione, esplicitare, in caso contrario, le eventuali ragioni di un tale comportamento. Nord (1991), inoltre, è dell'opinione che in una traduzione letteraria gli elementi "stranieri" dovrebbero essere visibili, cosa che non avviene nella traduzione delle opere per l'infanzia, dove, invece, si tende a sostituire nomi di personaggi, usi e costumi con elementi più vicini alla cultura del lettore. Seguendo queste indicazioni teoriche il corpus esaminato e tradotto dall'italiano al croato ha compreso sei poesie di Rodari. Facciamo presente che l'autore è stato tradotto soltanto nella prosa mentre nessuno finora ha osato tradurre i suoi versi. Le sei poesie sono state analizzate e discusse entro due filoni distinti e cognitivamente separati: quello del Rodari universale, la cui tematica è presente in tutte le culture a prescindere dalla lingua veicolare, e quello del Rodari locale, con caratteristiche tipiche del territorio e della cultura italiana. Per il primo filone abbiamo scelto le poesie Le parole, Pesi e misure e Speranza e per il secondo La testa del chiodo, Il povero ane e Filastrocca corta e matta.

\section{ANALISI DEI TESTI}

Se per traduzione intendiamo una pura e semplice transcodificazione linguistica, siamo concordi con gli autori che sostengono la tesi dell'intraducibilità della poesia (Folena 1994). Anche i poeti stessi appartenenti a differenti epoche e aree linguistiche hanno dichiarato l'impossibilità di mantenere nella traduzione il significato integrale di un testo poetico. Già Dante nel Convivio scriveva "nulla cosa per legame musaico armonizzata si può de la sua loquela in altra transmutare, sanza rompere tutta la sua dolcezza e armonia" (VII: 16) accennando alla violenza del processo traduttivo che spezza un legame armonico e frantuma un'unità non ricostituibile. Per la natura divina e profetica della poesia, la concezione del poeta come demiurgo nega implicitamente la possibilità di ricreare in un altro codice linguistico il messaggio poetico e vede nella traduzione (necessariamente traditrice) una profanazione dell'atto creativo. L'essenza del messaggio poetico è possibile mantenere solo attraverso un processo che ha la stessa intensità di quello originale (Bassnett 1993). D'altro canto, Steiner (2019) polemizzando contro i teorici dell'intraducibilità, dichiara che l'attacco alla traduzione è solo una debole forma di attacco al linguaggio stesso. "Il pregiudizio dell' intraducibilità viene automaticamente a cadere se si intende il tradurre non come ricerca di equivalenze semantiche o formali, ma nella sua più ampia accezione di attività linguistica che presiede a qualsiasi tipo di scambio comunicativo, fra lingue diverse o anche nell' ambito della stessa lingua" (Campanini 2002: 8). Ora, arrivando al merito di questo saggio, dob- 
biamo affermare che tradurre la letteratura per l'infanzia non è facile come potrebbe sembrare di primo acchito. Questo tipo di traduzione viene spesso sottovalutato, in quanto considerato semplice e poco importante. Difatti la traduzione deve tener conto della dualità narrativa, ovvero la difficoltà di dover attirare contemporaneamente il gruppo principale di destinatari, costituito dai bambini e quello secondario e autoritario, rappresentato dagli adulti (Puurtinen 1995). Puurtinen (1995) suggerisce a tal proposito che la sottovalutazione della letteratura per l'infanzia è dovuta al fatto che tratta argomenti apparentemente semplici e che secondo alcuni si basa su schemi ricorrenti in termini di struttura, personaggi e lingua.

Il pensiero poetico di numerose liriche di Rodari è fortemente legato alla sua terra e alla sua cultura, forse troppo palesemente ancorato all'ambiente locale e presuppone che il lettore conosca le problematiche messe in gioco: dai nomi dei personaggi ai nomi di montagne e città. Ci sono brani e poesie che palesano una grande conoscenza del territorio e che invita tutti a ridiventare abitanti del proprio posto, partendo dalla compilazione di una mappa personale del posto che includerà uno studio della vegetazione, degli animali, della geologia e della storia locale. Tutti questi elementi, nelle liriche di Rodari, riguardano un gruppo circoscritto di persone che appartengono alla cultura italiana e che comprendono le espressioni metaforiche, le abitudini linguistiche dei fiorentini, i falsi accrescitivi, i giochi linguistici. Analizzando superficialmente il testo si nota un continuo e costante utilizzo di lessemi specifici indicanti nomi, località, oggetti ben precisi e tangibili: il cortile, il Colosseo, Trastevere, Ponte Sisto, una miriade di nomi concreti e di persona. Il suo parlato è pieno di forme linguistiche delle parlate locali, immagina "conseguenze fantastiche determinate dagli errori di ortografia che hanno il potere di modificare la realtà" (Califano, 1998: 31) e così si crea una lingua in continuo rapporto con il lettore, un linguaggio vivace e vitale che racconta l'Italia.

L'altra faccia della sua poetica è rivolta al pensiero universale, alle tendenze universali, perché il desiderio di denunciare alcuni atteggiamenti, comportamenti e abitudini non è più solo limitato agli italiani, ma a tutti gli uomini, all'intera umanità. Sono idee e concetti generali e astratti applicabili a tutti gli individui, leggi universalmente accettate: il valore e il significato delle parole, i pesi e le misure, la speranza. Le sue parole sono chiare, pacifiste, dette senza ambiguità. Questo è il messaggio universale della sua opera che dimostra anche una grande ricchezza ed eleganza poetica. Il pacifismo, l'antimilitarismo, l'internazionalismo, l'uguaglianza sociale, il lavoro, i diritti fondamentali degli uomini, tutti ricavati dall'esperienza pratica dello scrittore, occupano molte pagine delle opere di Rodari e vengono rappresentati come ideali universali e indispensabili per la salvezza di una Terra ancora troppo malata. 


\subsection{Rodari universale}

Presentano gli elementi dell'universalità delle liriche rodariane, passiamo all'analisi delle soluzioni traduttive e le diverse proposte quali segnali di specifiche poetiche e politiche traduttive. Ogni traduzione contiene una visione personale dell'attraversamento/superamento dei confini linguistici e culturali, e questi approcci possono essere particolarmente chiari dal modo in cui sono stati tradotti gli antroponimi che in Rodari sono marcati semanticamente (Grgić Maroević \& Roić 2019).

\begin{tabular}{|l|l|}
\hline Le parole & Riječi \\
Abbiamo parole per vendere & Imamo riječi za prodaju \\
parole per comprare & riječi za kupovinu \\
parole per fare parole & riječi koje tvore riječi \\
ma ci servono parole per pensare. & al' trebamo i riječi za razmišljanje. \\
Abbiamo parole per uccidere & Imamo riječi za ubojstvo \\
parole per dormire & riječi za spavanje \\
parole per fare solletico & riječi za škakljanje \\
ma ci servono parole per amare. & al' trebamo i riječi za ljubav. \\
Abbiamo le macchine & Imamo strojeve \\
per scrivere le parole & za pisanje riječi \\
dittafoni, magnetofoni & diktafone, snimače \\
microfoni & mikrofone \\
telefoni. & telefone. \\
Abbiamo parole & Imamo riječi \\
per fare rumore, & za stvaranje buke, \\
parole per parlare & riječi za govor \\
non ne abbiamo più. & nemamo ih više. \\
& \\
\hline
\end{tabular}

Il secondo libro delle filastrocche (Rodari 1985: 3)

Il secondo libro delle filastrocche di Gianni Rodari comprende filastrocche che mettono in evidenza la libertà dei bambini. Questo libro prosegue idealmente il cammino delle notissime Filastrocche in cielo e in terra con divertenti trovate in rima. Il primo problema traduttologico nel quale siamo incappati è la traduzione dei verbi italiani che in croato possono venir riprodotti in due modi:

1. usando i sostantivi provenienti dai verbi, ossia i nomi verbali (in croato glagolske imenice: prodavanje, kupovanje, stvaranje, ubijanje, voljenje, govorenje) dato che l'ortografia croata non permette l'uso di per $[z a]+$ verbo all'infinito (za prodati, za kupovati, za stvarati, ecc.). Ci sono casi in cui il sostantivo verbale è un nome derivato da un verbo e ha tutte le proprietà di un nome e nessuna proprietà del verbo (leggere-lettura; uccidere-uccisione). 
2. usando i sostantivi veri e propri (prodaju, kupovinu, ubojstvo, govor). Nel caso specifico della traduzione di questa poesia abbiamo optato per la seconda possibilità traduttiva ritenendola più consona alla lingua croata. In tal caso abbiamo però dovuto rinunciare alla rima. Laddove non era possibile usare i sostantivi veri e propri ci siamo ancorati alla prima soluzione decidendo per i sostantivi provenienti dai verbi.

Rodari termina le prime due strofe con una sentenza apostrofata con $m a$, congiunzione avversativa che in croato viene ripresa con al' (ali con troncamento) per mantenere il ritmo recitativo. Le parole Diktafone, magnetofone // mikrofone // telefone [dittafoni, magnetofoni // microfoni // telefoni] potrebbero evocare la stessa omofonia dell'originale italiano. Rimaniamo perplessi sul termine magnetofone che in croato è ormai una parola desueta e ci sembra logico sostituirla con quella oggi in uso snimač (nell'accusativo snimače). Questa poesia è stata tradotta avvalendoci di ambedue le opportunità grammaticali offerte dalla lingua croata che denotano due proposte diverse a seconda del gusto estetico personale (Pistone 2001).

\begin{tabular}{|c|c|c|c|}
\hline Pesi e misure ${ }^{4}$ & & Utezi i mjere & \\
\hline Non puoi pesare in metri & A & Ne možeš vagati u metrima & A \\
\hline né il pane né il panettone, & B & Ni kruh ni slastice fine & B \\
\hline né misurare in litri & A & ni mjeriti u litrama & A \\
\hline l'altezza del Resegone ... & B & visinu planine... & $\mathrm{B}$ \\
\hline Non compri a chili la stoffa & - & Ne kupuješ tkaninu na kilograme & - \\
\hline se vuoi farti il cappotto: & B & želiš li si sašiti kaput: & $\mathrm{B}$ \\
\hline non si vendono a ettari & - & ne prodaju se u hektarima & - \\
\hline i funghi per il risotto. & B & gljive za rižot. & $\mathrm{B}$ \\
\hline Lo so, tu non confondi & - & Znam, ti ne brkaš & - \\
\hline i pesi e le misure: & B & utege i mjere nikad: & B \\
\hline $\begin{array}{l}\text { sei del sistema metrico } \\
\text { un gran campione ... Eppure ... }\end{array}$ & & u metričkom sustavu & \\
\hline un gran campione ... Eppure ... & B & ti si prvak ... Ali ponekad... & B \\
\hline Nel misurare gli uomini & - & U mjerenju ljudi & - \\
\hline puoi sbagliare anche tu: & B & može pogriješiti svatko: & $\mathrm{B}$ \\
\hline il più ricco, il più forte & - & nabogatijeg, najsnažnijeg & - \\
\hline li stimi un po'di più... & B & naučiti više cijeniti prilično je lako... & $\mathrm{B}$ \\
\hline$C^{\prime}$ è chi misura il prossimo & - & Ima onih koji mjere bližnjeg & - \\
\hline magari dal suo colore, & B & možda po njegovoj boji, & B \\
\hline mentre dell'uomo conta & - & dok kod čovjeka važnost & - \\
\hline non la pelle, ma il cuore. & B & ne koža, već srce kroji. & $\mathrm{B}$ \\
\hline
\end{tabular}

${ }^{4}$ Testo disponibile sul sito: https://www.poesie.reportonline.it/poesie-di-gianni-rodari/ poesia-di-gianni-rodari-pesi-e-misure. html [Consultato il 10/11/2020]. 
Nel verso puoi sbagliare anche tu Rodari si rivolge a ognuno di noi personalmente, elemento che viene mantenuto nella traduzione možeš i ti pogriješiti. Volendo invece portare il messaggio a un livello più universale si potrebbe optare per može pogriješiti svatko [tutti possono sbagliare], frase maggiormente esortativa. Lo stesso ragionamento può venir fatto per le varianti malo si više naučio cijeniti [li stimi un po' di più], versione personalizzata, rispetto a naučiti više cijeniti prilično je lako [imparare a rispettare di più è piuttosto facile], versione più impersonale, quindi tendente a diventare generalizzata, quasi una tesi moralistica. L'aver scelto la traduzione più universale, collettiva, rivolta a tutti è un atteggiamento giustificato come anticipazione del messaggio universale e proverbiale di Rodari presente nell'ultima strofa. Oggi numerosi bambini stanno vivendo un dramma senza fine quello del pericoloso rischio di denutrizione, freddo, malattie, perdita di familiari, migrazioni forzate, morti, amputazioni, mancanza di ogni diritto basilare, in primis il loro "diritto alla vita e al gioco". Con la consueta sensibilità ed eleganza egli tratta un tema estremamente delicato: il razzismo.

Panettone e Resegone non sono termini significativi per la comprensione della poesia, benché siano stati scelti del poeta (molto probabilmente) per creare la rima, fattore non indifferente quando si parla di poesia. Ciò nonostante, una minima sostituzione dei termini non cambia il valore e il senso delle due parole. È possibile che la parola panettone sia stata inserita da Rodari per il suono che rinvia a "pane" (presente nello stesso verso), ma non troviamo ragioni plausibili per non sostituirlo ad es. con "focaccia". Similmente, il lessema Resegone (ossia nome di una montagna, poco conosciuta ai più) riunisce in sé alcuni semi di "spazialità", "dimensionalità", "verticalità". L'identificazione di queste tre unità minime di contenuto ci autorizza a trovare un altro sostantivo (nome proprio o comune) avente gli stessi atomi di significato, questa volta più vicini alla sfera culturale dei giovani lettori. In questo verso abbiamo preferito riprendere il nome comune di montagna [planina] mantenendo per di più la rima dell'originale. Un'altra possibile soluzione sarebbe quella di conservare il nome panettone essendo ormai un dolce conosciuto un po' in tutto il mondo, ma accettando per forza il nome di Resegone che non è una montagna conosciuta ai bambini croati. In tal caso si dimostrerebbe utile aggiungere la parola $v r h$ [cima] come pars pro toto o sineddoche di montagna. 


\begin{tabular}{|l|c|l|c|}
\hline Speranza & & Nada & \\
S'io avessi una botteguccia & - & Kad bih imao dućančić & - \\
fatta d'una sola stanza & B & koji stane u jednu sobu & B \\
vorrei mettermi a vendere & - & htio bih prodavati & - \\
sai cosa? La speranza. & B & znaš što? Nadu. & B \\
«Speranza a buon mercato!» & - & "Nadu nudim povoljno!" & - \\
Per un soldo ne darei & B & Za jednog bih gosta & B \\
ad un solo cliente & - & za novčić dao & - \\
quanto basta per sei. & B & koliko je za šest dosta. & B \\
E alla povera gente & & & \\
che non ha da campare & - & A siromasima & B \\
darei tutta la mia speranza & - & koji nemaju od čega živjeti & - \\
senza farla pagare. & B & kao bih svu svoju nadu & B da ništa ne moraju platiti. \\
\hline
\end{tabular}

Filastrocche in cielo e in terra (Rodari 1972: 87)

Questo stile liscio, semplice, versatile, facilmente comprensibile deve rimaner tale anche nella traduzione croata. A fare da padrone è il messaggio forte che traspare da ogni singolo verso e si materializza (come di consueto in Rodari) sull'ultimo verso dove vive il suo apogeo, un assunto di una morale condivisa. L'esortazione a non arrendersi mai e a tenere sempre alta la speranza sono doti e qualità che dovrebbero animare ogni persona, esser accessibili a tutti e dunque "a buon mercato". Rodari ha saputo dare, con un'umanità indescrivibile, voce a chi di voce, spesso, ne aveva poca.

\subsection{Rodari locale}

Tradurre i versi di Rodari ai bambini croati vuol dire andare oltre la pura corrispondenza tra parole delle due lingue diverse: si deve entrare nel mondo infantile di cultura croata e comprenderlo tenendo presente le abilità cognitive e la maturità emotiva di fruitori con cultura diversa da quella italiana. Inoltre, è necessario scegliere un lessico parimenti interessante e vivace, che offre ai giovani destinatari una forte motivazione alla lettura, ma anche la possibilità di crescere creativi, inventivi e di approcciarsi a un mondo fantastico spesso lasciato in disparte. 


\begin{tabular}{|c|c|c|c|}
\hline La testa del chiodo & & Glava čavla & \\
\hline La palma della mano & - & Ruke, dlanovi & A \\
\hline$i$ datteri non $f a$, & B & nisu planovi, & A \\
\hline sulla pianta del piede & - & nitko se neće popeti na taban & B \\
\hline chi si arrampicherà? & B & ali hoće li ipak netko na tavan? & $\mathrm{B}$ \\
\hline Non porta scarpe il tavolo, & - & Stol ne nosi cipele & - \\
\hline su quattro piedi sta: & B & Na četiri se noge naslanja: & B \\
\hline il treno non scodinzola & - & vlak repom ne maše & - \\
\hline ma la coda ce l'ha. & B & a rep ima. & B \\
\hline $\begin{array}{l}\text { Anche il chiodo ha una testa, } \\
\text { però non ci ragiona: }\end{array}$ & $\overline{\mathrm{B}}$ & $\begin{array}{l}\text { I čavao ima glavu, } \\
\text { al' njome ne razmišlja, ne: }\end{array}$ & $\overline{\mathrm{B}}$ \\
\hline la stessa cosa càpita & - & istome su pak & - \\
\hline a piú d'una persona. & B & i neke osobe sklone. & B \\
\hline
\end{tabular}

Filastrocche in cielo e in terra (Rodari 1972: 15)

Il linguaggio di Rodari semplice e diretto, ironico e delicato descrive emozioni e sentimenti e insegna ai bambini le cose importanti della vita, permettendo loro di arrivare alla profondità delle cose vere. "La palma della mano" e "la pianta del piede" sono termini metaforici che come tali una volta tradotti perdono quell'immagine mentale che si crea nella lingua originale. Difatti la forma femminile 'palma' si riferisce all'albero dei datteri da cui derivava etimologicamente (De Mauro \& Mancini 2000: 1437). Pianta, dal latino planta (De Mauro \& Mancini 2000: 1522), è un nome generale che comprende tutti i vegetali che espandono in aria i rami e le foglie. Per questo motivo il significato si estende anche sulla parte piatta e larga di sotto il piede inferendogli le caratteristiche di fondamenti e stabilità come le radici di una pianta. Questa caratteristica semantica, dove una parola ha due significati, si perde nella traduzione in croato che riproduce i due concetti non con valori metaforici ma con semplici sostantivi: palma della mano vs. palma - dlan vs. palma; pianta del piede vs. pianta vegetale - taban vs. biljka. Una possibilità traduttiva potrebbe essere quella di ricorrere alla salvaguardia dell'assonanza (fattore non trascurabile nella poesia) mediante parole che conservano una corrispondenza fonetica e lo stesso ritmo come nel caso di: dlanovi/planovi e taban/tavan. In tal caso però nuovamente abbiamo sacrificato l'impostazione generale di Rodari che gioca sul doppio senso del significato delle parole. Volendolo assecondare la traduzione anche in questa doverosa esigenza, proponiamo di sostituire i primi quattro 
versi (improponibili in croato nel format originale) inserendo altre parti del corpo umano come uši e kosa che in croato hanno due significati diversi:

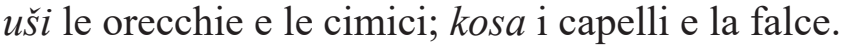

\begin{tabular}{|l|l|l|c|}
\hline Il povero ane & & Jadan Ane & \\
Se andrete a Firenze & - & Ako idete u Firencu & \\
vedrete certamente & - & vidjet ćete gore & - \\
quel povero ane & - & tog štenca Ane & $\mathrm{B}$ \\
di cui parla la gente. & $\mathrm{B}$ & o kom ljudi zbore. & - \\
E un cane senza testa, & - & On je pas bez glave, & $\mathrm{B}$ \\
povera bestia. & $\mathrm{B}$ & zvijer kojom se bave. & - \\
Davvero non si sa & - & Stvarno se ne zna & $\mathrm{B}$ \\
ad abbaiare come fa. & $\mathrm{B}$ & kako lajati uspije. & - \\
La testa, si dice, & - & Glavu, & $\mathrm{B}$ \\
gliel'hanno mangiata... & $\mathrm{B}$ & pojeli mu, kažu... \\
(La " c " per i fiorentini & - & (Slovo «k» firentinci & - \\
è pietanza prelibata). & $\mathrm{B}$ & u slast smažu) & $\mathrm{B}$ \\
Ma lui non si lamenta, & - & Al' on se ne žali & - \\
è un caro cucciolone, & $\mathrm{B}$ & štene je ljupko & $\mathrm{B}$ \\
scodinzola e fa festa & - & maše repom i slavi & - \\
a tutte le persone. & $\mathrm{B}$ & i žensko i muško. & $\mathrm{B}$ \\
Come mangia? Signori, & - & Kako jede? Gospodo, \\
non stiamo ad indagare: & $\mathrm{B}$ & nećemo istraživati: & - \\
ci sono tante maniere & - & postoji mnogo načina & $\mathrm{B}$ \\
di tirare a campare. & $\mathrm{B}$ & kako preživljavati. & - \\
Vivere senza testa & - & Živjeti bez glave & $\mathrm{B}$ \\
non è il peggio dei guai: & $\mathrm{B}$ & Nije najgora stvar: & - \\
tanta gente ce l'ha & - & toliko je ljudi ima & $\mathrm{B}$ \\
ma non l'adopera mai. & $\mathrm{B}$ & al' da je koristi bar. & - \\
& & & $\mathrm{B}$ \\
\hline
\end{tabular}

Il libro degli errori (Rodari 1964: 20)

Il libro degli errori ci porta in un universo linguistico disordinato e scomposto in cui il magico Rodari trasforma in divertimento i noiosi esercizi grammaticali e ogni svista linguistica, muta in gioco le regole della nostra grammatica aprendo un dialogo fitto e ricchissimo con i lettori. La scelta della filastrocca Il povero ane è stata una vera sfida traduttiva. Vediamo in ordine le presupposizioni dalle quali siamo partiti per giungere a una chiave di lettura logica da poter applicare alla traduzione.

${ }^{5}$ In italiano ane è una versione abbreviata della parola cane. Nella traduzione la parola viene usata come il nome proprio dell'animale. 
Il titolo poteva venir tradotto nei seguenti modi: "Jadan rek", "Jadan uko" o "Jadan as", come abbreviazione della parola cane a seconda del nome attribuitogli nelle diverse parti della Croazia: in Istria "brek", in Slavonia "ćuko" e nelle regioni dove si parla lo standard (pas). Questo nel caso si ritenesse corretto mantenere l'idea iniziale del poeta di "toglierli la testa". Ovviamente i tre tentativi di tradurre il termine dell'animale sono fallimentari in quanto le regioni che usano i tre termini non hanno l'abitudine di tralasciare, trascurare, nella pratica orale, la prima lettera.

Un'altra strada da percorrere potrebbe essere quella di trovare un'altra parola, un altro animale, che inizia con una lettera che realmente in qualche parte della Croazia si ha l'abitudine di saltare o semplicemente pronunciare in maniera differente dallo standard. Ovviamente una tale soluzione mette a dura prova la traduzione che non sarà più tale, ma si tratterà di versione in quanto il testo verrà adattato alla situazione concreta vissuta dai parlanti croatofoni. In tal caso il rinvio a Firenze e alla pronuncia toscana della $c$ aspirata, ovvero della gorgia, si perderà del tutto.

Una terza possibilità è quella di lasciare il testo come un lembo di terra extraterritoriale che racconta la storia linguistica dei parlanti fiorentini. La traduzione tiene conto delle lacune culturali e linguistiche dei lettori croatofoni e ne avvicina il contenuto e il messaggio ricorrendo a una spiegazione a pie' di pagina: la parola ane deriva dalla parola cane ma le viene omessa la "c" iniziale, come conseguenza della caratteristica fonica dei Toscani.

\begin{tabular}{|l|c|l|l|}
\hline Filastrocca corta e matta & & Brojalica kratka i šašava & \\
Filastrocca corta corta 8 & & Arojalica kratka kratka 8 & \\
Il porto vuole sposare la porta, 11 & A & A \\
la viola studia il violino 9 & A & Vrat želi oženiti vrata svaka, 11 & A \\
il mulo dice: - Mio figlio è il mulino -; 13 & B & viola violinu uči svirati 9 & B \\
la mela dice: - Mio nonno è il melone -,12 & C & medo kaže: - Moja će se kći Svjećica zvati -; 13 & B djed zove Medenjak -, 12 \\
il matto vuole essere un mattone 11 & C & C \\
e il più matto della terra 9 & ovaj šaš pak želi biti šašav čak 11 & C \\
sapete che vuole? Fare la guerra. 11 & D & a najluđi na svoj planeti 9 & D \\
& D & znate li vi što želi? Ratovati. 11 & D \\
\hline
\end{tabular}

Prime fiabe e filastrocche (Rodari 2011: 60)

Il quarto e il quinto verso della poesia nell'originale propongono delle soluzioni ritmiche comprese nelle false parole alterate, diminutivi e accrescitivi (viola/violino; mulo/mulino, mela/melone, matto/mattone). Le soluzioni in croato sono di diversa natura in quanto si tratta di parole derivate (svijet) svjećica; medo/medenjak; šav/šašav) con l'intento di mantenere lo stesso morfema lessicale. Nel secondo verso le parole porto/porta aventi lo stesso morfema lessicale diventano maschile o femminile a seconda del morfema 
grammaticale $-O$ oppure $-a$ aggiunto. In croato abbiamo scelto le parole vrat/vrata che, pur non avendo lo stesso significato semantico (collo/porta), rendono lo stesso effetto sonoro. Un caso a parte è la corrispondenza dei termini viola/violino mantenuti nella traduzione in croato in quanto termini internazionali di strumenti musicali. Interessante in questa filastrocca è il fatto che la rima AABBCCDD, la lunghezza del verso e l'assonanza nella traduzione croata sono stati rispettati in assoluto.

\section{CONCLUSIONE}

Mossi dall'impulso di rendere nella lingua croata qualcosa di fortemente allettante e stimolante in italiano, abbiamo deciso di tradurre Gianni Rodari, per la sua fantasia sfrenata che crea innumerevoli e inimmaginabili provocazioni traduttive. La sua delicata ironia, il linguaggio diretto e tuttavia denso di significati, i giochi di parole, le rime, le riflessioni scanzonate sulla lingua e sugli errori che possono dar luogo a mondi inaspettati, sono un'ulteriore sfida per il traduttore che ha bisogno di perizia, fantasia e grande sensibilità per ricreare nella propria lingua il fitto intreccio di significati e musicalità dell'originale ${ }^{6}$. La traduzione di queste poesie deve rimanere ugualmente interessante, vivace, ironica, deve far ridere, e quindi non comporta solo la traduzione interlinguistica, ma richiede anche l'adattamento del testo alla cultura e all'età del giovane lettore. Partendo dal presupposto che "Tradurre vuol dire situarsi tra le lingue. Stare tra le lingue. Rispondere alla parzialità di ogni lingua con un passaggio di confine" (Prete 2011: 12), il nostro problema stava esattamente nel mezzo tra una lingua e l'altra, esplicitato tra "traduzione neutralizzante" e "traduzione straniante" da un lato, e "belle infedeli" e "brutte fedeli" dall'altro (Rossi \& Sofo 2015: 12). Analizzando e traducendo una piccola, ma esemplificativa parte delle opere di Rodari abbiamo dovuto risolvere una a una ciascuna di queste sfide. Solo tramite un lavoro traduttivo dettagliato, sodo e leale possiamo offrire ai bambini croati l'opportunità di imparare ad apprezzare la cultura italiana, da un lato, e diventare più tolleranti e aperti verso una cultura diversa dalla loro, dall'altro (Garavini 2014).

${ }^{6}$ Reperibile su: https://www.punto-italiano.it/gianni-rodari-eventi-podcast-iniziative-per-il-maestro-della-fantasia [Consultato il 12/12/2020]. 


\section{BIBLIOGRAFIA}

Alighieri, D. (1988). Convivio. In C. Vasoli \& D. De Robertis (a cura di), Opere minori (tomo I, parte II). Torino: U.T.E.T.

Bassnett, S. (1993). La traduzione. Teorie e pratica. Milano: Bompiani.

Boero, P. (2007). Ah, come sono belle, certe volte, le cose sbagliate. In F. Lullo \& T. V. Viola (a cura di), Il cavaliere che ruppe il calamaio (pp. 13-24). Novara: Interlinea.

Buongiorno, T. (1995). Dizionario della letteratura per ragazzi. I personaggi, le trame, i temi d'attualità. Milano: A. Vallardi, Garzanti.

Califano, F. (1998). Lo specchio fantastico. Realismo e surrealismo nell'opera di Gianni Rodari. Trieste: Edizioni EL.

Campanini, S. (2001). Dinamica traduttiva di un testo poetico: The Departing Island di Iain Crichton Smith. In G. Parks (a cura di), Traduzione poetica e dintorni (pp. 99-109). Trieste: Università degli studi di Trieste.

Campanini, S. (2002). Strategie e metodi della traduzione poetica. L'Harmattan Italia: Torino.

De Mauro, T. \& Mancini, M. (2000). Garzanti etimologico. Milano: Garzanti linguistica.

Denti, R. (2014). Le fiabe sono vere. Novara: Interlinea.

Ewers, H.-H. (2009). Fundamental Concepts of Children's Literature Research: Literary and Sociological Approaches. New York and London: Routledge.

Folena, G. (1994). Volgarizzare e tradurre. Torino: Einaudi.

Formicola, C. (2015). Tradurre poesia! Tradurre poesia? Bollettino di studi latini, XLV, 1, 92-111. Napoli: Paolo Loffredo editore.

Garavini, M. (2014). La traduzione della letteratura per l'infanzia dal finlandese all'italiano: l'esempio degli albi illustrati di Mauri Kunnas. Turku: Annales universitatis turkuensis.

Grgić Maroević, I. \& Roić, S. (2019). Gianni Rodari: organičko i tehničko kao etičko. Detinjstvo Childhood, 44 (2), 11-16.

Nord, C. (1991). Scopos, Loyalty, and Translation Conventions. Target 3 (1), 91-109.

Palermitano, A. (2020). Gianni Rodari in altre lingue, 14/05/2020. Testo disponibile sul sito https://www.newitalianbooks.it/it/gianni-rodari-nel-mondo/ [Consultato il 15/11/2020].

Pistone, M. (2001). Uso dei nomi verbali. L'infinito. In Lingua italiana. Sintassi (parte prima, capitolo XX). Testo disponibile sul sito https:// www.mauriziopistone.it/testi/sintassi/ capitolo120.html [Consultato il $10 / 12 / 2020]$.

Praz, M. (1925). Poeti inglesi dell'Ottocento. Firenze: Bemporad. 
Prete, A. (2011). All'ombra dell'altra lingua. Per una poetica della traduzione. Torino: Bollati Boringhieri.

Puurtinen, T. (1995). Linguistic Acceptability in Translated Children's Literature. Joensuu: University of Joensuu.

Rei $\beta$, K. \& Vermeer, H. J. (1984). Grundlegung einer allgemeinen Translationtheorie. Tübingen: Niemeyer.

Reynolds, K. (2011). Children's Literature: A Very Short Introduction. Oxford: Oxford University Press.

Rodari, G. (1972). Filastrocche in cielo e in terra. Torino: Einaudi.

Rodari, G. (1964). Il libro degli errori. Torino: Einaudi.

Rodari, G. (1985). Il secondo libro delle filastrocche. Torino: Einaudi.

Rodari, G. (2011). Prime fiabe e filastrocche. San Dorligo della Valle, Trieste: Edizioni EL.

Rossi, G. \& Sofo G. (2015). Sulla traduzione. Itinerari fra lingue, letterature e culture. Chieti: Solfanelli.

Scotellaro, F. (2020). Da Rodari a Pavićević, le sfide di tradurre letteratura per l'infanzia. Intervista a Marija Spasić e Elisa Coppetti alla Ca' Foscari di Venezia, 9/6/2020. Testo disponibile sul sito https://www. unive.it/pag/14024/?tx_news_pi1\%5Bnews\%5D=9039 \&cHash= 680603700ed2d091c1274f4409366b96 [Consultato il 22/10/2020].

Shavit, Z. (1980). The Ambivalent Status of Texts: The Case of Children's Literature. Poetics Today, vol. 1 (3), Special Issue: Narratology I: Poetics of Fiction, 75-86. Duke University Press. Testo disponibile sul sito https://www.jstor.org/stable/i303048 [Consultato il 7/4/2021]. Steiner, G. (2019). Dopo Babele. Aspetti del linguaggio e della traduzione. Milano: Garzanti.

Vermeer, H. J. (1996). A Skopos Theory of Translation (Some Arguments for and against). Heidelberg: TextconText.

Zalar, D. (2011). Gianni Rodari. In V. Višković (a cura di), Hrvatska književna enciklopedija. (vol. 3, p. 589). Zagreb: Leksikografski zavod Miroslav Krleža.

\section{Sitografia}

https://it.wikipedia.org/wiki/Gianni_Rodari

https://www.poesie.reportonline.it/poesie-di-gianni-rodari/poesia-di-gianni-rodari-pesi-e-misure.html

https://www.punto-italiano.it/gianni-rodari-eventi-podcast-iniziative-per-il-maestro-della-fantasia

https://it.wikipedia.org/wiki/Premio_Hans_Christian_Andersen 


\section{GIANNI RODARI: WORDS TO CONSIDER, WORDS TO LOVE}

\section{Summary}

The dilemmas that have always tormented those who deal with poetic translation can be summarized in two basic questions: the first, of a predominantly philosophical nature questioning the possibility to translate poetry, and the second, of a practical nature, questioning how to translate a poetic text (Campanini 2001). This essay offers an attempt of poetic translation of some nursery rhymes/poems by Rodari, from Italian into Croatian. The translation is accompanied by some functional reflections, without pretending to propose an ideal model, since "the quality of a translation cannot be established a priori" (Praz 1925: 10), but it can only have a fortuitous validity, being linked to the tastes of the specific customs of a time period or to the aesthetic and ideological values of an individual translator. This means that what will count more than critical approval is the consent and willingness of the vast and heterogeneous public to express a judgment of acceptance or rejection. The dynamics of the linguistic and conceptual operations that generated the final version of these translations was very complex and discussed considering that, in making of the individual translation choices, it was necessary to simultaneously take into account multiple and different factors in their interaction: content, rhythm, rhymes, assonances, meanings, customs, culture.

Keywords: Gianni Rodari, poetic translation, globalism, locality, culture and tradition. 